\title{
Cricket Inspired Flow-Sensor Arrays
}

\author{
Gijs Krijnen, Theo Lammerink, Remco Wiegerink \\ Transducers Science \& Technology group \\ MESA+ Research Institute, Univeristy of Twente \\ Enschede, The Netherlands \\ gijs.krijnen@utwente.nl
}

\author{
Jérôme Casas \\ Institut de Recherche sur la Biologie de l'Insecte \\ IRBI UMR CNRS 6035, Université François Rabelais \\ Tours, France
}

\begin{abstract}
We report current developments in biomimetic flow-sensors based on mechanoreceptive sensory hairs of crickets. These filiform hairs are highly perceptive to lowfrequency sound with energy sensitivities close to thermal threshold. In this work we describe hair-sensors fabricated by a combination of sacrificial poly-silicon technology, to form silicon-nitride suspended membranes, and SU8 polymer processing for fabrication of hairs with diameters of about 50 $\mu \mathrm{m}$ and up to $1 \mathrm{~mm}$ length. The membranes have thin chromium electrodes on top forming variable capacitors with the substrate allowing for capacitive read-out. Previously these sensors have been shown to exhibit acoustic sensitivity. Based on a hydrodynamic - mechanical interaction model we derive a figure of merit. We present optical measurements on acoustically excited hair-sensors. Experimental data and the derived models are shown to exhibit good correspondence.
\end{abstract}

\section{INTRODUCTION}

In comparing biological and engineered systems for similar functions one often finds striking differences in implementation; for example when taking a look at auditory sensing. 1) In biological systems sensing elements are based on the flow-sensitivity of large arrays of parallel operating mechano-sensors (hairs, cilia). 2) Biological systems rely heavily on mechanical filtering and amplification. And 3) noise may play a beneficial role when perceiving signals near the noise limits due to the nonlinear properties of the sensors. Engineered systems for acoustic sensing on the other hand are based on pressure measurements using single moving structures (e.g. membranes as in microphones), perform filtering and amplification in the electronic domain (in sequential manner), and generally see their usable dynamic sensing range limited by noise. An example of mechanical filtering in biology is found in the auditory system of mammals where tapered "sound-board" like resonator structures (basilar membrane) with complex interacting inner- and outer- hair-cells perform distributed filtering, amplification and adaptation in the mechanical domain [1]. At the same time parallelism helps to overcome constraints of bandwidth of the neural systems and provides robustness, redundancy and gradual decline. Examples of how biological systems benefit from noise are seen in the form of stochastic resonance and amplification [2] as observed in crickets [3] and crayfish [4]).

This research was made possible by grants from the Customized Intelligent Life-Inspired Arrays project funded by the Future and Emergent Technologies arm of the IST Programme and by the BioEARS Vici grant of the Dutch Technology Foundation (STW/NWO).

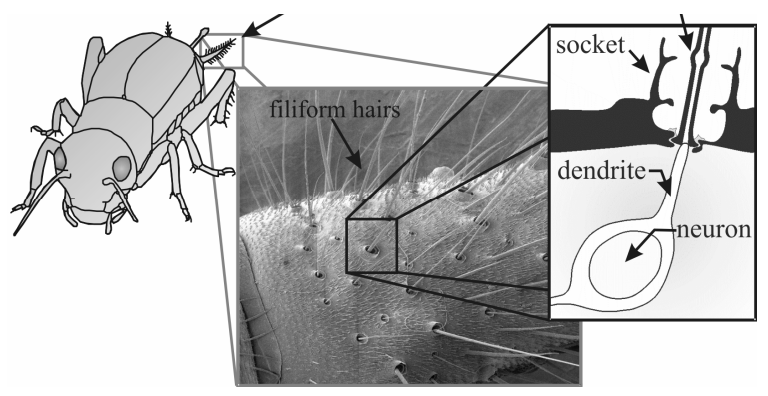

Figure 1. Filiform hairs on the cerci of crickets [8].

Despite the advancement of mankind in many areas of technology it is still challenging for engineered systems to compete with biological systems. For example: the auditory capabilities of bats to perceive their environment, locate prey and to navigate at high velocities through complex surrounding (e.g. with leafed brushes and trees) has no manmade equivalent. Likewise the sensitivity of hair-based acoustic mechano-sensors, found on insects [5], to perceive acoustic signals at thermal noise levels is astounding. It is this kind of performance that raises interest in biological systems with the purpose to gain insight in entirely new principles or in order to improve engineered systems and gain extended functionality.

In biomimetics researchers seek to take inspiration from nature for the purpose of making engineered artifacts either fulfilling a set task or as a model-system for further study in a biological context. Evidently, the study of nature requires a trained biologist whereas modeling and fabrication is best done by physicists and engineers. Hence, ideally biologists and engineers work together addressing mutual topics and over a longer period to allow for iterations that facilitate depth and width of understanding and optimization of the engineered structures. Along with many colleagues, the authors of this paper have had the privilege to work together on cricked sensory hairs for the last five years by virtue of two consecutive European Union framework programs: Cricket Inspired perCeption and Decision Automata (Cicada, [6] and Customised Intelligent Life-Inspired Arrays (Cilia, [7]). 
Our research was initially motivated from the notion under biologists that the sensory hairs of crickets ${ }^{1}$ are amongst the most sensitive sensors in nature displaying energy sensitivities at the level of thermal fluctuations (kT) [5]. What makes these sensory hairs even more interesting is that they generally are found in large numbers on the body of the animals, especially grouped on the two elongated appendices at the abdominal end of the body, the so-called cerci. Hair densities depend considerably on the position on the cerci but may be as high as 400 per $\mathrm{mm}^{2}$ locally [8]. Arraying of directional sensitive sensory hairs has allowed crickets to acoustically image their environment with high angular resolutions [9]. Furthermore one can speculate about variability in hair-sizes, geometry and suspension which in theory (but not experimentally proven) could allow crickets to perceive sound frequency resolved.

It seems a viable proposition to use MEMS technology to mimick these sensors since MEMS allows for fabrication of large arrays of hairs at the scales comparable to the cricket sensory hairs. Clearly, if artificial sound/flow sensors could tap a fraction of the potential of the cricket sensory system it would be worthwhile investigating this proposition. It could yield low frequency sensitive flow sensors applicable to measurement of particle velocity associated with sound. Arrays of these sensors would enable flow field imaging with high densities, hardly conceivable with e.g. hot wire based anemometric sensors. It could be a stepping stone towards frequency resolved sound measurements akin to cochlear sound decomposition and perception. In more general terms it would allow for temporally, frequency and spatially resolved measurements and facilitate the recognition of specific sound/flow signatures, much alike the cricket sensory hair system is supposed to play a major role in the detection of the approach of predators like spiders and wasps, see figure $2[8,10]$.

\section{BIOMIMETICS OF CRICKet INSPIREd HAIR-SENSORS}

In understanding a certain biological locomotion or sensory system many insights need to come together before one can even start thinking in terms of biomimicry. E.g. to understand sensory systems physiological data is required to get a first understanding of the possible operation principle. This data has to be complemented by materials properties since many mechanical properties (stiffness, density, anisotropy, etc.) can cause distinctive variations in (multimodal) sensitivities. For example, air flow sensitive hairs are long and thin and have low moment of inertia, low mass and low stiffness sockets. Comparable hair-sensors but with much shorter, thicker and heavier hairs will be less flow sensitive due to boundary layer effects (see below) but may be optimized to operate as accelerometer sensors. Clearly the flow sensitive hairs will show some acceleration sensitivity as well but the overall structure determines its maincharacteristic as being flow-sensitive. Putting shape and materials together may suffice to gain physical insight into

\footnotetext{
${ }^{1}$ These exquisite sensitivities are not only found on crickets but on
} many arthropods e.g. also on cockroaches and spiders. the sensory system and allow for (mechanical) response modeling. A next step to understand the sensory system is the neural transduction part, i.e. how is the mechanical response translated into a neural spike signal. Since this transduction is inherently nonlinear it is amenable to such effects as stochastic resonance $[3,4,5]$ and parametric effects [11]. Further complicating the matter in arrayed systems, such as the air-flow sensitive hairs of crickets, the entire canopy response is used in the "neural processors" (the terminal ganglion and higher neural centers). These neural centers do not necessarily only integrate the signals from one sort of sensor but may integrate a variation of sensor modalities. Clearly, this information processing should enable the detection of specific sensory signatures that signify e.g. danger (the approach of a predator). Unraveling the neural circuitry is a formidable task and much is still unknown. Although each of the parts of this chain of information sensing, transduction, transport, processing and signature detection may be separately understood to some extend, only the complete chain has biological meaning since natural selection eliminates the whole organism and not some of its constituents. For example, if the hair-sensors allow for high frequency flow sensing, but the neural system is incapable of coding these signals, the overall performance of the system may be non-optimal. This is why in sensory ecology biologists try to determine the main evolutionary pressures and the sensory tasks following from these pressures. Most importantly in this respect are the predator prey relations. I.e. predation pressure in combination with evolutionary developments will give important clues to taskbased optimization of sensory systems. It is in the context of this optimization that all parts of the "sensing - perception action" chain can be evaluated for their contribution to the overall efficacy of the system. It implies that one can try to

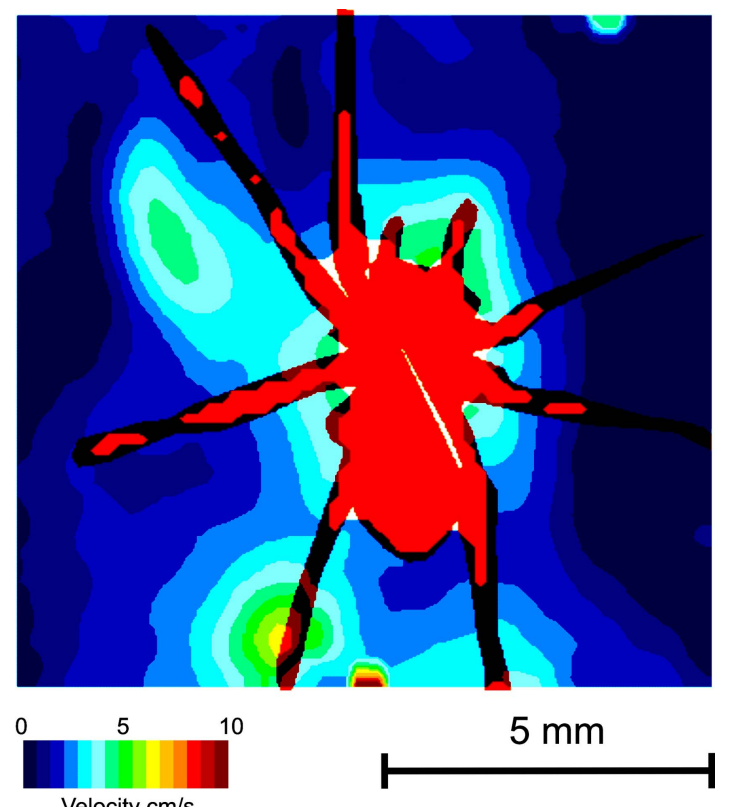

Velocity $\mathrm{cm} / \mathrm{s}$

Figure 2. Transients air displacement generated by a walking spider as observed by Particle Image Velocimetry [12]. 
understand the sensory response in relation to the ecological relevant stimuli. In other words, one tries to identify the reasons why these very crickets have such sensitive hairs; what is so special in their life which requires such a degree of finesse?

In the example of the crickets of this work one of us (JC and his group) has conducted extensive research on the interaction between wood crickets (Nemobius sylvestris) and the wolf spider (Pardosa, Lycosidae). Part of this research consists of quantifying the air movements generated by spiders and acting as stimuli to the cricket hair-sensors during a spider attack [12] (see figure 2). Evidently, also flows over the crickets' cerci need to be quantified [13] to understand the hydrodynamic interactions. With each step of sophistication in the understanding of the hair-sensory system, as natures optimizations to tackle certain tasks unfold, the chance for successful biomimetic implementation improves. At the same time it may become clear that nature's specific optimization is not necessarily the optimization the engineer is looking for but with the gained knowledge, translated into appropriate design rules, "bio-copying" may be truly turned into "bio-inspiration".

\section{HAIR BASED FLOW SENSING}

The sensory hairs of the cricket are situated on the back of the body on appendices called cerci. Depending on species, the hairs of adults vary in length up to $1.5 \mathrm{~mm}$. E.g. for Nemobius sylvestris and Gryllus bimaculatus bimodal distributions are found with concentrations around $150 \mu \mathrm{m}$ and $750 \mu \mathrm{m}[14,15]$. Each hair is lodged in a socket, guiding the hair to move in a preferred direction and held in place by an elastic material surrounding the base. Airflow causes a neuron to fire, by rotation of the hair base (figure 1). Depending on hair-length and frequency sensitivities can be down to 10 's of $\mu \mathrm{m} / \mathrm{s}$ flow velocity amplitude [5].

It is interesting to note that crickets have developed flow sensitive hairs rather than pressure sensitive sensors. As Tautz has pointed out [16] this is more or less a necessity

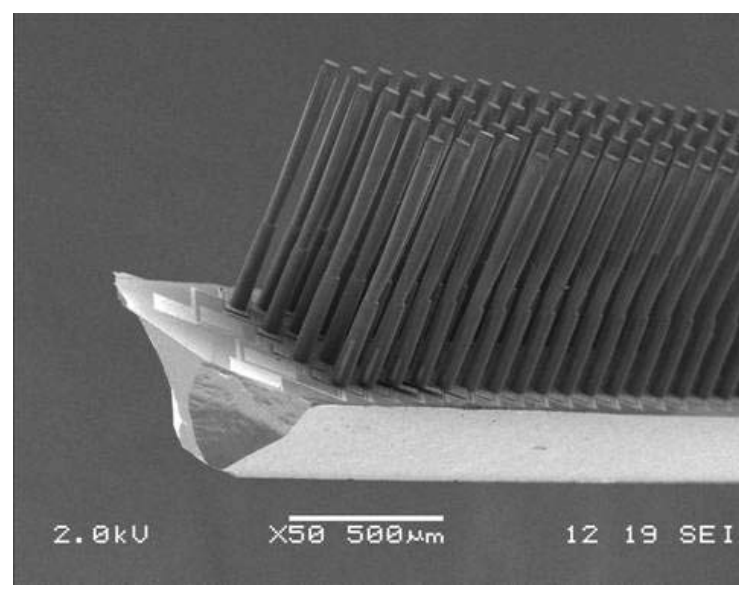

Figure 3. Scanning Electron Microscope image of actual sensors. The electrical connection to the top-electrodes runs over the torsion beams

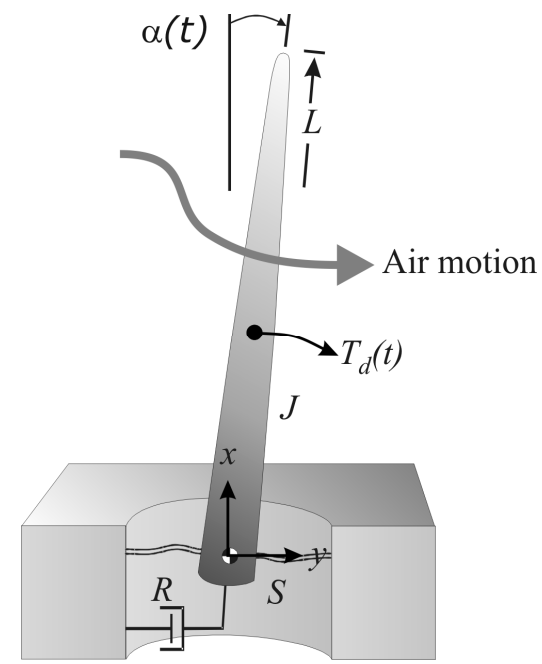

Figure 4. Hair-sensors can be modeled as inverted pendula representing damped second order mechanical systems (adapted from [14]).

since important predators of crickets, such as wasps and spiders, are too small to produce any significant pressure variations at the important frequencies of e.g. wing beats (tens to hundreds of $\mathrm{Hz}$ ). This observation is based on the fact that substantial pressure waves can only be emitted by moving bodies larger than $\approx 2 \pi \lambda$, with $\lambda$ the wavelength of the sound. Moreover, many attacks on crickets will take place at relative short distances such that the cricket may be considered in the near field of the sound producing predator, i.e. where particle velocity is more readily conceivable than pressure fluctuations. Moreover, as the approaching object is very near, it would be preferable to escape in the right direction, information that is concealed in the particle velocity but not in the pressure field.

\section{A. Mechanical system}

Here we will briefly reiterate the theoretical framework developed for the understanding of flow-sensitive mechanosensors of crickets $[17,18]$ and apply it to our biomimetic sensors. A filliform hair is described as an inverted pendulum, a second order mechanical system, determined by the torsional spring constant $S$, the moment of inertia $J$ and the torsional resistance $R$ (figure 4 ). Conservation of angular momentum requires that

$$
J \frac{d^{2} \alpha(t)}{d t^{2}}+R \frac{d \alpha(t)}{d t}+S \alpha(t)=T_{d r a g}(t)
$$

where $T_{\text {drag }}$ is the flow induced drag torque. Indicative values for cricket filiform hairs with a length of $1000 \mu \mathrm{m}$ are: $S \approx$ $2 \cdot 10^{-11} \mathrm{Nm} / \mathrm{rad}, \mathrm{J} \approx 5 \cdot 10^{-18} \mathrm{~kg} \cdot \mathrm{m}^{2}$ and $\mathrm{R} \approx 10^{-14} \mathrm{Nms} / \mathrm{rad}$ [17]. Resonance frequencies of filiform hairs are in the range of 30 to $3000 \mathrm{~Hz}$ depending on hair-length and structural allometric scaling [17]. However, best frequencies are lower since the systems are moderately damped and have almost constant quality factor over the entire range of hair-length. The damping is hypothesized to have evolved under constraints of impedance matching to the mechanical 
impedance of the driving flow as given by the Stokes expressions [5]. Impedance matching allows for maximum energy transfer, hence optimized sensitivity of the sensory hairs.

For harmonic excitation torque at radial frequency $\omega$ the steady oscillatory hair movement $\alpha(\omega)$ can be described by the well-known complex amplitude function:

$$
\alpha_{0}(\omega)=\frac{T_{d 0}(\omega)}{J} \cdot \frac{1}{\omega_{0}^{2}-\omega^{2}+j \omega(R / J)}
$$

where $T_{d 0}(\omega)$ is the amplitude of the drag-torque and $\omega_{0}$ is the resonance frequency given by $(S / J)^{0.5}$.

\section{B. Aerodynamic modeling}

The hairs are deflected by viscous drag on the hair shaft due to the particle velocity. For a harmonic flow velocity parallel to a flat surface given by a far-field of the form

$$
v_{y, \infty}(t)=V_{0} \cdot \sin (\omega t)
$$

the frequency dependent velocity profile above the surface is given by $[17,18]$ :

$$
\begin{aligned}
& v_{y}(x, t)=V_{0} \cdot \sin (\omega t)-V_{0} \cdot e^{-\beta x} \cdot \sin (\omega t-\beta x) \\
& \beta=\sqrt{\frac{\omega}{2 \cdot v}}
\end{aligned}
$$

with $v$ the kinematic viscosity, $x$ the distance from the surface and $\omega$ the angular frequency of the harmonically oscillating flow. Due to viscosity and the assumption of noslip at the interface between fluid and fixed surface there is a transition zone between zero velocity and the far-field velocity, the so-called boundary layer. The boundary layer thickness $\left(\delta_{b}\right)$ depends on $2 / \beta$, being larger at lower frequencies. Figure 5 shows calculated and measured normalized flow-profiles above flat surfaces for various time-instants of one period of oscillation. Agreement between both is excellent permitting to use (4) in further modeling approaches.

In the small Reynolds number regime the drag-forces can be described by the Stokes expressions. These state that the drag-force by a fluid-flow given by (3) on a cylinder of unit length is given by [17]:

$$
\begin{aligned}
F_{d}(t)= & \left\{4 \pi \mu \cdot \frac{-g}{g^{2}+(\pi / 4)^{2}}\right\} V_{0} \cdot \sin (\omega t)+ \\
& \omega\left\{\pi \rho_{a} a^{2}+\frac{4 \pi \mu}{\omega} \cdot \frac{\pi / 4}{g^{2}+(\pi / 4)^{2}}\right\} V_{0} \cdot \cos (\omega t)
\end{aligned}
$$

with $\mu$ the dynamic viscosity, $a$ the radius of the cylinder and $\rho_{a}$ the mass-density of air. The variable $g$ is given by:

$$
g=\ln (a \beta / \sqrt{2})+\gamma
$$

where $\gamma$ is Euler's constant $(0.5772 .$.$) .$
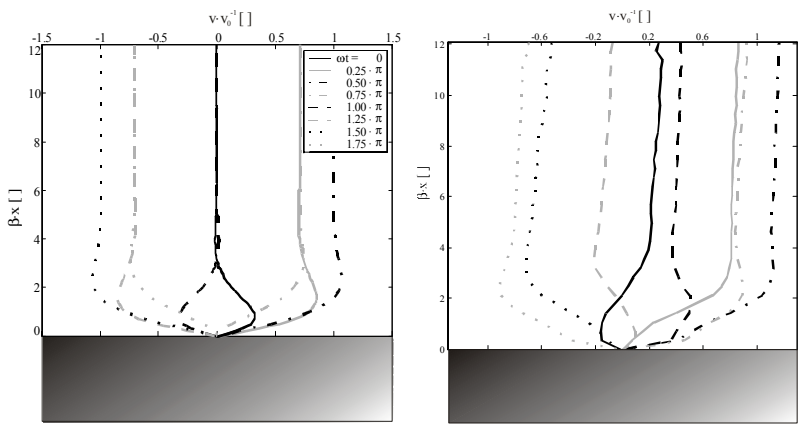

Figure 5. Oscillating boundary layer on a flat surface, a) calculated, b) measured using a Particle Image Velocimetry setup, normalised with: $v_{\text {air }}=1.79 \cdot 10^{-5} \mathrm{~N} \cdot \mathrm{s} \cdot \mathrm{m}^{-2}, v_{0}=3 \mathrm{~cm} \cdot \mathrm{s}^{-1}$ and $\mathrm{f}=60 \mathrm{~Hz}$.

The velocity in (5) is actually the velocity difference between the flow and the hair. However, the stiffness of our hairs is on the order of $10^{-8} \mathrm{Nm} / \mathrm{rad}$ and hair movement is relatively small in our structures so that the velocity difference can be approximated by the flow velocity itself (eq. 3). The total torque on the filiform hairs can be calculated by integrating the drag-moment along the hair:

$$
T_{d}=\int_{0}^{L} F_{d}(x) x d x
$$

Using (4), (5) and (7) the total drag-torque on the hairs can be determined. The integration (7) is readily done
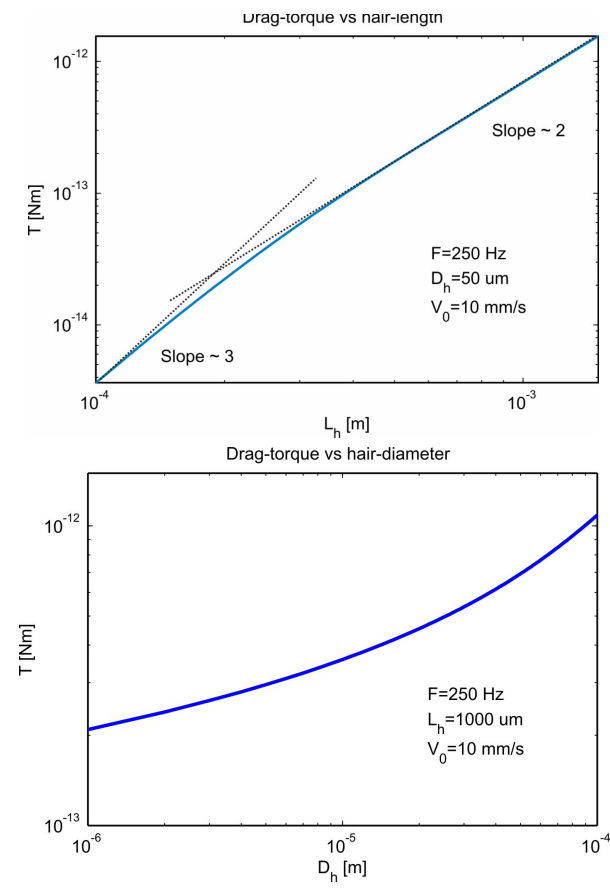

Figure 6. Drag-torque predictions by evaluation of (7) at $250 \mathrm{~Hz}$ in air. Top: Drag-torque acting on a cylinder of $50 \mu \mathrm{m}$ diameter, as a function of hair length. Bottom: Drag-torque acting on a $1 \mathrm{~mm}$ long cylinder as a function of hair diameter. 


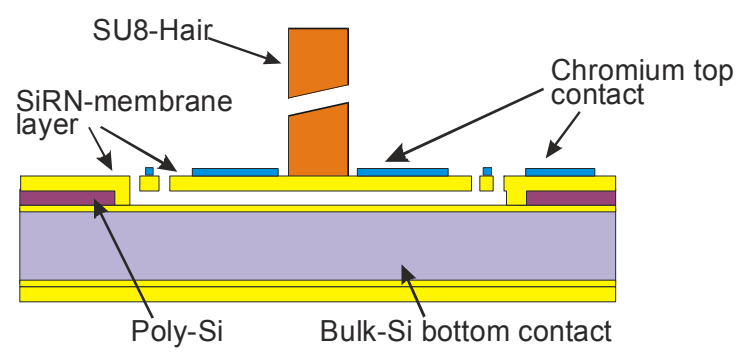

Figure 7. Schematic representation of artificial biomimetic flowsensors based on cricket mechano-sensory hairs.

numerically.

The drag-torque increases roughly proportional to the hair length cubed $\mathrm{L}^{3}$ when $\mathrm{L}<2 / \beta$ showing the importance of hair-length for optimized sensitivity (see figure 6). For a hair to effectively pick-up drag-torque it is essential that it extends out of the boundary layer. With a kinematic viscosity of $15.1 \cdot 10^{-6} \mathrm{~m}^{2} / \mathrm{s}$ for air and at a frequency of $100 \mathrm{~Hz}$ this translates into a minimum hair-length of about $440 \mu \mathrm{m}$. For lower frequencies this length is even longer. Once the hairlength is larger $\mathrm{L}>2 / \beta$ the drag-torque increases proportional to the length squared, as can be expected from the constant velocity amplitude (4) and (5) and (7). The drag-torque amplitude of a $1 \mathrm{~mm}$ long, $50 \mu \mathrm{m}$ diameter hair, exposed to a harmonic air flow of $250 \mathrm{~Hz}$ was calculated to be $6 \cdot 10^{-11} \mathrm{Nm}$ per $\mathrm{m} / \mathrm{s}$ of flow-velocity amplitude as an indication of the torque-magnitude that can be expected. Varying the hair diameter is not dramatically affecting the drag-torque, especially not at low frequencies, as can be seen from figure 6 but also from (5).

\section{Artificial hair-sensors}

Artificial mechano-sensory hairs (figures 3 and 7) are based on the tilting of a membrane due to viscous drag. The membrane is suspended by torsion beams. In the sensors described here both the membrane and the torsion beams are made of silicon nitride. The membranes can have various forms, e.g. circular or rectangular. Chromium electrodes are deposited on top of the membrane. These electrodes form capacitors with the underlying common electrode formed by the highly doped silicon wafer. Due to the drag-induced torque the membranes tilt and therefore the capacitors on both halves of the sensor will show (opposite) change in capacitance. These changes in capacitance can be determined differentially and provide a means of measuring the tilting angle and, hence, the flow causing the tilt. Fabrication of these sensors is described elsewhere $[19,20]$. An example of a "semi-cercus" like sensor array is shown in figure 3 .

\section{Transducer modeling}

The sensitivity $(\eta)$ to measure drag-induced rotation is proportional to the change in capacitance per unit rotation. Since one half of the membrane moves upward and the other downward, the capacitance difference per unit of rotation can be calculated in the parallel plate approximation from:

$$
\eta=\left.2 \cdot \frac{\partial C}{\partial \alpha}\right|_{\alpha=0}=2 \cdot \lim _{\alpha \rightarrow 0} \frac{\partial}{\partial \alpha}\left(\int_{A} \frac{\varepsilon_{0}}{d_{0}-\alpha y} d A\right)=2 \varepsilon_{0} \int_{A} \frac{y}{d_{0}^{2}} d A
$$

where use has been made of $\sin \alpha \approx \alpha$ for the small angles of rotation encountered, in which $y$ is the distance to the rotation axis, $A$ is the area of half of the membrane. The dielectric thickness $d_{0}$ is given by:

$$
d_{0}=d+\frac{t_{1}+t_{2}}{\varepsilon_{r}}
$$

where $\varepsilon_{0}$ and $\varepsilon_{\mathrm{r}}$ are the dielectric constant of air and the relative dielectric constant of silicon nitride, respectively. Sensors have been fabricated with circular as well as with rectangular membranes. Using (8) $\eta$ can be easily calculated for rectangular and circular membranes. Typically the gap between the electrodes $(d)$ is $1 \mu \mathrm{m}$ and the thickness of the silicon nitride layers $t_{1}$ and $t_{2}$ are $1 \mu \mathrm{m}$ and $0.1 \mu \mathrm{m}$ respectively. In case of circular membranes $R$ is $100 \mu \mathrm{m}$ giving a sensitivity of $\eta_{C}=9.0 \mathrm{pF} / \mathrm{rad}$ whereas in case of rectangular membranes $w=200 \mu \mathrm{m}$ and $1=100 \mu \mathrm{m}$ giving a sensitivity of $\eta_{R}=15.4 \mathrm{pF} / \mathrm{rad}$.

\section{E. Performance predictions}

Using the result of the drag torque calculations $(3-7)$ in the mechanical response expression (20) model predictions can be made regarding the sensor's mechanical performance. Geometric and other values are listed in table 1. Comparing artificial hair-sensors with cricket sensory hairs of about 1 $\mathrm{mm}$ length it is interesting to note that a) the rotational stiffness of the MEMS sensors is about 360 times higher than that of the crickets and b) the moment of inertia is about 150 times larger. This translates into a 360 times smaller sensitivity and about 1.6 times higher resonance frequency (see figure 11).

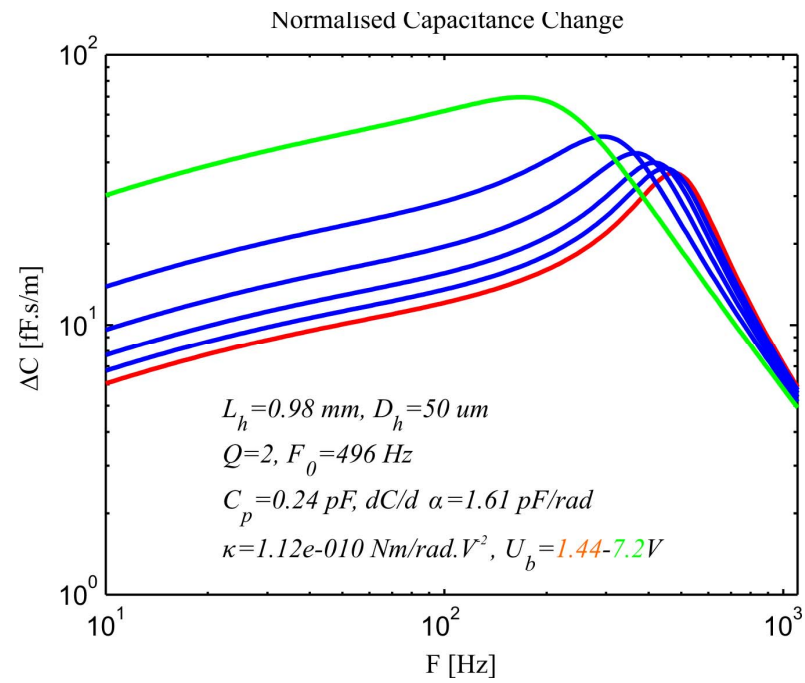

Figure 8. Sensor-response model-predictions including the effect of electrostatic spring softening (see secion V). 
TABLE I.

SENSOR DIMENSIONS AND MATERIAL PROPERTIES USED FOR THE MODEL PREDICTIONS

\begin{tabular}{|l|r|l|r|l|r|}
\hline Membrane (SiRN) & & Springs (SiRN) & & Hairs (SU-8) & \\
\hline Diameter & $210 \mu \mathrm{m}$ & Length & $75 \mu \mathrm{m}$ & Length & $980 \mu \mathrm{m}$ \\
\hline Thickness & $0.9 \mu \mathrm{m}$ & Width & $10 \mu \mathrm{m}$ & Diameter & $50 \mu \mathrm{m}$ \\
\hline Gap spacing & $1 \mu \mathrm{m}$ & Thickness & $0.9 \mu \mathrm{m}$ & Mass density & $1200 \mathrm{~kg} / \mathrm{m}^{3}$ \\
\hline Curvature $\delta$ & $2.0 \mu \mathrm{m}$ & Youngs Modulus & $310 \mathrm{GPa}$ & & \\
\hline Diameter electrodes & $200 \mu \mathrm{m}$ & Poisson ratio & 0.24 & & \\
\hline$\varepsilon_{\mathrm{r}}$ & 7.5 & & & & \\
\hline \hline Sensitivity $\eta_{C}$ & $1.39 \mathrm{pF} / \mathrm{rad}$ & Stiffness $S$ & $7.2 \cdot 10^{-9} \mathrm{Nm} / \mathrm{rad}$ & Inertial Moment & $0.744 \cdot 10^{-15} \mathrm{~kg} \cdot \mathrm{m}^{2}$ \\
\hline
\end{tabular}

Figure 8 shows various curves for a range of effective suspension stiffnesses. Since the mechanical model does not include any physical damping, e.g. such as squeezed film effects, the damping coefficient $R$ has been set to produce a quality factor $Q=2$ at the highest spring stiffness (red curve). This results in a decrease of quality factor with increasing effective spring stiffness (decreasing resonance frequency). Multiplying the calculated rotations by the sensitivity $\eta$ of the sensors the predicted sensor response is on the order of $10-100 \mathrm{fF} \cdot \mathrm{s} / \mathrm{m}$ and these values can be scaled up linearly with the number of hair-sensor in parallel. However, important to note is that the actual measurement resolution depends on the ratio of capacitance change to nominal capacitance value [20]. This implies that large parasitic capacitance, as often encountered in MEMS sensors, will deteriorate the sensor resolution and sensitivity.

\section{F. Figure of Merit}

In order to optimize the hair-sensors and to allow a comparison between artificial and natural hair-sensors we make the following observations. a) When the torsional spring stiffness is low the drag-induced rotations are large providing high sensitivity. Hence a low rotational stiffness seems desirable. b) The resonance frequency of the hairs depends on the torsional stiffness $S$, the lower $S$ the lower the resonance frequency $\omega_{0}$ at given moment of inertia $J$. It is not unreasonable to take the resonance frequency $\omega_{0}$ as a measure for the bandwidth in which the sensors can be used since above this frequency the response decreases with 12 $\mathrm{dB} /$ octave. c) Longer hairs do experience larger drag-torque than smaller hairs (figure 6, top). The drag-torque increases approximately proportional to the hair length cubed $\left(L_{h}\right)^{3}$, when $L_{h}$ is smaller than the boundary layer thickness $\left(\delta_{b}\right)$ and with approximately $\left(L_{h}\right)^{2}$ when $L_{h}>\delta_{b}$. Hence long hairs seem to be desirable for high sensitivity. d) At the same time hair-length $L$ occurs cubed in the inertial moment $J$ having a large impact on $\omega_{0}$. e) The diameter of the hairs has a quadratic influence on the inertial moment, but only a very slight effect on drag-torque (figure 6, bottom). Although depending on frequency, in the frequency range of interest the relation between the drag-torque and the diameters can be approximated by $\propto\left(D_{h}\right)^{1 / 3}$.

Sensor-optimisation has (at least) two dimensions: usable bandwidth and (low-frequency) sensitivity. If one of both is (too) low the usability of the sensor is strongly hampered. We have also argued that both sensitivity and bandwidth depend strongly on $S, L$ and $D_{h}$. Therefore we define a figure of merit (FOM) as the product of usable bandwidth (i.e. proportional to $\omega_{0}$ ) and sensitivity (i.e. the drag-torque to which a hair is exposed divided by the rotational stiffness). Using the above simplifications we arrive at:

$$
F O M \equiv \omega_{0} \cdot \frac{T_{d}}{S}=\sqrt{\frac{S}{J}} \cdot \frac{T_{d}}{S} \propto \sqrt{\frac{S}{\rho L_{h}^{3} D_{h}^{2}}} \cdot \frac{L_{h}^{2} D_{h}^{1 / 3}}{S}=\sqrt{\frac{L_{h}}{\rho S D_{h}^{4 / 3}}}
$$

This FOM shows that sensitive sensors with a large usable bandwidth should have long, thin hairs made of low density material, and small torsion stiffness, exactly what is seen in nature. In comparing the FOM of the artificial hairsensors to the FOM of the crickets' hair-sensors, we find that cricket hairs perform about a 100 times better mechanically (for $1 \mathrm{~mm}$ long hairs). Crickets out-perform artificial sensors because of their low torsional stiffness $\left(2 \cdot 10^{-11}\right.$ vs. $7 \cdot 10^{-9}$ $\mathrm{Nm} / \mathrm{rad}$ ) and small average hair-diameter (4.5 vs. $50 \mu \mathrm{m})$.

\section{EXPERIMENTAL}

In a previous paper [21] we have used capacitive characterization to demonstrate acoustic sensitivity and figure-ofeight directional sensitivity. Here we show measurements on acoustic mechanical sensitivity by means of Laser Vibrometry (Polytec MSA400). These measurements give us the advantage of increased sensitivity and resolution and allow for direct observations of the acoustically induced tilt-angles, hence providing a means to compare measurements with aerodynamic-mechanical models. The measurement set-up is schematically shown in figure 9 .

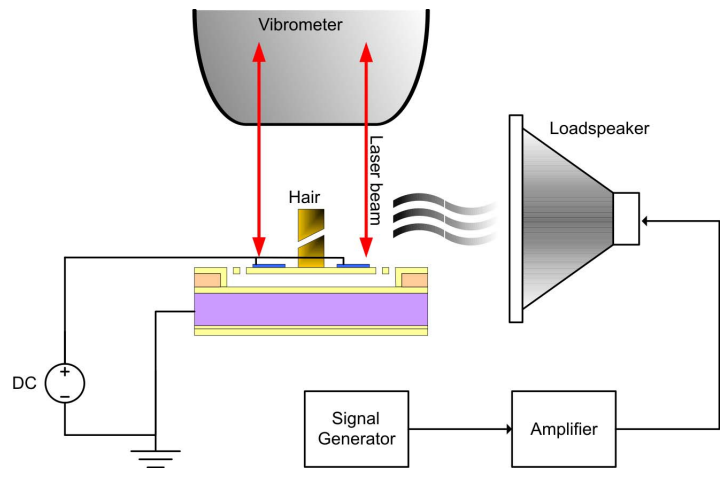

Figure 9. Schematic of the measurement setup using a loudspeaker as very-near field sound source and a laser-vibrometer for detection of out-of-plane displacements at the rim of the membrane 
The sensors are positioned under a scanning laservibrometer at a distance of a about $5-10 \mathrm{~mm}$ from a square silicon plate $\left(\approx 2.5 \times 2.5 \mathrm{~cm}^{2}\right)$ mounted on a loudspeaker. Since the distance of the hairs to the plate is so small, the loudspeaker induced particle velocity at the position of the hairs is in good approximation equal to the velocity of the loudspeaker itself. For a characteristic dimension of the sound source $L$ this so-called very-near field regime [22] exists at distances from the source smaller than $L / 2 \pi$ and if $L$ is much smaller than the wavelength $\lambda$. Since we are interested in relative low frequencies $(<1.0 \mathrm{kHz}, \lambda / 2 \pi>5.4$ $\mathrm{cm}$ ) the assumption of a very-near field source holds for distances of less than about $6 \mathrm{~mm}$.

The scanning laser beam of the LV was adjusted to the rim of the membranes at the largest distance from the rotational axis. Since this distance was know, the displacements amplitudes were readily converted to angles of rotation which are comparable to the model predictions. The obtained rotational angles were divided by the velocity amplitudes of the silicon plate at given generator-signals and frequencies, measured separately with the LV. This yields the normalized angular rotation which is displayed as a function of frequency in figure 10. The model parameters were chosen in accordance to the device design and using readily available material properties as listed in Table 1 . The only exception to this is the damping which was chosen to provide a quality factor of 2, roughly fitted to the experimentally observed transfer-function.

Comparison of the modeled and experimentally obtained data shows very satisfying similarity, not only qualitatively but also with respect to normalized rotation angles, hence giving the model credibility as well as the design optimizations derived from the model.

\section{DISCUSSION}

The previous sections have shown that the theory developed as a framework for the understanding of flowsensing in crickets by filliform hairs [17, 18] is highly

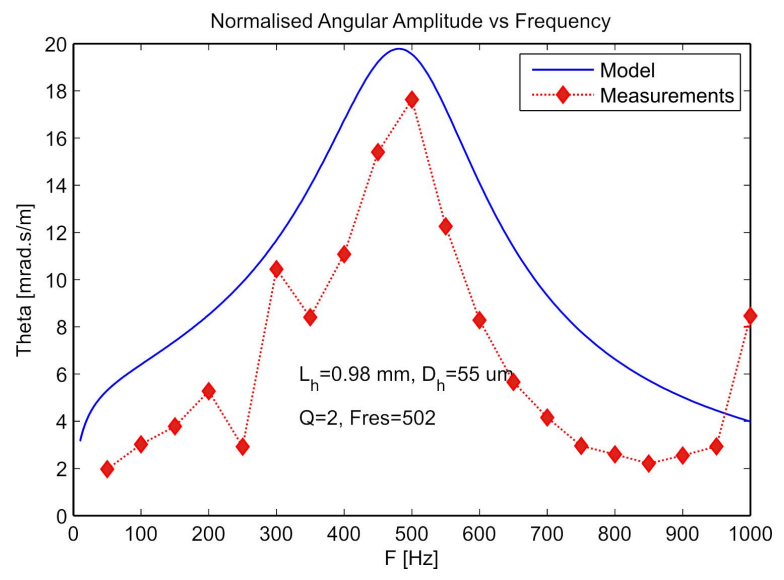

Figure 10. Angular rotation normalized to $1 \mathrm{~m} / \mathrm{s}$ flow-velocity amplitude as obtained from the measurements and as predicted by the model. applicable to artificial hair-sensors. In comparison to crickets the structures made by MEMS technology seem rather well defined and also the materials parameters are mostly wellknown. Moreover, more complicating effects like viscoelastic damping certainly occurring in the form of squeezed film damping in-between the capacitor plates, do not seem to obscure the physical first order effects, i.e. the mechanical second order nature of the artificial hair-sensors is clearly revealed in the experimental observations.

What needs to be emphasized is that the sensitivity of the artificial sensors is about 2.3 orders of magnitude smaller than the hair-sensors of equal length in crickets hence rotation angles are much smaller implying that higher order effects are not easily observed. Looking at figure 11 it is clear that further optimization is required and as mentioned earlier the defined FOM gives clear directions for optimization with crickets scoring high with respect to this FOM.

One of the attractive features of crickets' flow-sensitive hair-sensors is their high energy-sensitivity. In order for biomimetic hair-sensors to get to similar sensitivities much work still needs to be done.

Changing the drag-torque pick-up. The FOM introduced in section 2 shows that larger tilting angles at a usable bandwidth can only be obtained by using thin, long hairs, mounted on compliant suspensions. This way the inertial moment is kept low while the drag-torque does not suffer considerably. For our sensory hairs this implies that we need to look at increasing the aspect-ratio of the hair structures. The SU-8 currently used provides aspect ratios of about 10 . Other shapes, e.g. by stacking two dissimilar parts, may partly resolve the problem. However, as far as mass-density goes, SU-8 is comparable in density at about $1.24 \cdot 10^{3} \mathrm{~kg} / \mathrm{m}^{3}$ [23] to the cuticle out of which cricket hairs are made with a density of about $1.2 \cdot 10^{3} \mathrm{~kg} / \mathrm{m}^{3}$ [24]. Making hairs of comparable size with smaller inertial moment may potentially be achieved by using porous materials.

Towards flexible rotation. For drag-torques to produce large tilts evidently small rotational stiffness is required. However, in the current sensor design the torsion beams do not only provide angular compliance $(S)$ but also have a limited stiffness $(K)$ in vertical ( $x$-direction in figure 4). The governing equations for $S$ and $K$ show that $S$ is proportional to $L^{-1}$ and $K$ to $L^{-3}$ where $L$ is the length of the torsion beams. Hence on reducing $S$ by increasing $L, K$ will decrease faster than S. This has three detrimental effects: a) on decreasing $K$ the voltage at which vertical pull-in occurs may become impractically low (since the capacitors are interrogated using voltages of about $1 \mathrm{~V}$ or more), b) low $\mathrm{K}$ values may cause various stiction problems and c) in the dynamic response the resonance frequency of the vertical mode may get near the resonance frequency of the torsional mode causing unwanted mode-coupling. 


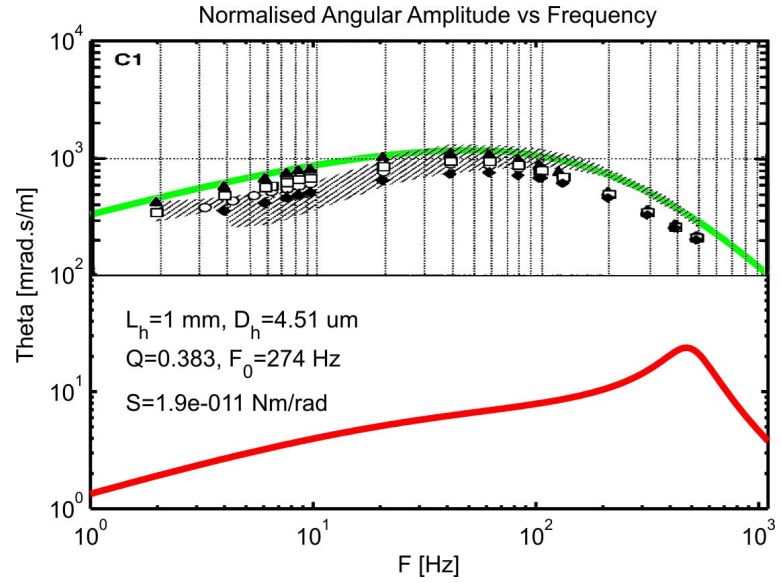

Figure 11. Comparison of measured cricket hair-deflection response $800-900 \mu \mathrm{m}$ long hairs (markers) with model predicted cricket hair response (green line) and artificial hair-sensor response (red line). Data from Shimozawa et al [17], normalized to $1 \mathrm{~m} / \mathrm{s}$ flow velocity amplitude.

\section{Perspectives}

In engineering biomimetic hairsensors one not necessarily has to limit oneself to "copying nature". Naturally, the organism and the engineer seldom have the same task to solve in their daily lives and since artificial implementations are (necessarily) based on different structures, additional limitations and possibilities may present themselves. E.g. using the capacitive read-out in our artificial hair-sensors allows for electrostatic actuation. Using a DC-bias voltage the effective spring stiffness can be adapted at will (see figure 8 and $[19,25]$ ) allowing for sensitivity modulation and frequency focusing. Other possibilities are the use of parametric effects [11], stochastic resonance [3] and cochlear inspired frequency resolved measurements. Most importantly, up to now we have made arrays in which all sensors were wired in parallel. Clearly one of the future tasks is to individually address the hair-sensors [20] and work on flow pattern measurements. Hence, having functional artificial hair-sensors at hand is only a starting point for further sensory research that will benefit both biologists and engineers.

\section{ACKNOWLEDGEMENT}

The authors want to thank: M. de Boer and E. Berenschot for their advice on processing, D. Altpeter for SU-8 processing, M. Dijkstra for the SEM pictures and our colleagues in the EU project CILIA for stimulating discussions.

\section{REFERENCES}

[1] K. Snyder et. al, F. Sachs, W. Brownell, Chapter 6 in "Sensors and Sensing in Biology and Engineering", ed. Barth, Humphry and Secomb (Springer) Vienna, 2003.

[2] T. Wellens, V. Shatokhin and A. Buchleitner, "Stochastic Resonance", Rep. Prog. Phys. 67, 2004.
[3] J. Levin, J. Miller, Nature, "Broadband neural encoding in the cricekt cercal sensory system enhanced by stochastic resonance", Vol 380, 1996, 165-168.

[4] S. Bahar, F. Moss, "Stochastic resonance and synchronization in the crayfish caudal photoreceptor", Mathematical Biosciences Vol. 188, 2004, 81-97.

[5] T. Shimozawa, J. Murakami, T. Kumagai, Chapter 10 in "Sensors and Sensing in Biology and Engineering", ed. Barth, Humphrey and Secomb (Springer), Vienna, 2003.

[6] Cicada: http://www.bionics-cicada.org/.

[7] Cilia: http://www.bionics-cilia.org/.

[8] O. Dangles, C. Magal, D. Pierre, A. Olivier, J.Casas, "Variation in morphology and performance of predator-sensing system in wild cricket populations", J. Exp. Bio. vol-208, pg 461-468, 2005.

[9] Landolfa M.A. and Jacobs G.A. "Direction sensitivity of the filiform hair population of the cricket cercal system", Journal of Comparitive Physiology A 177, 1995, 759-766

[10] O. Dangles, J. Casas and I. Coolen, "Textbook cricket goes to the field: the ecological scene of the neuroethological play", J. Exp. Bio. vol-209, pg 393-398, 2006.

[11] Carr D W, Evoy S, Sekaric L, Craighead H G and Parpia J M 2000 "Parametric amplification in a torsional microresonator", Appl. Phys. Lett. vol-77, pg 1545-1547, 2000.

[12] Unpublished results by J. Casas, T. Steinmann and coworkers

[13] T. Steinmann, J. Casas, G. Krijnen and O. Dangles, "Air-flow sensitive hairs: boundary layers in oscillatory flows around arthropod appendages" J. Exp. Bio. vol-209, 4398-4408, 2006.

[14] C. Magal, O. Dangles, P. Caparroy, J. Casas, "Hair canopy of cricket sensory system tuned to predator signals", J. Theor. Bio. 241 (2006) 459-466.

[15] O. Dangles, D. Pierre, C. Magal, F. Vannier and J. Casas, "Ontogeny of air-motion sensing in cricket”, J. Exp. Bio., vol 209, pg 4363-4370, 2006.

[16] J. Tautz, Naturwissenschaften, "Reception of particle oscillation in a medium- an unorthodox sensory capacity", Naturwissenschaften 66 , pg. 452-461, 1979.

[17] T. Shimozawa, T. Kumagai, Y. Baba, "Structural scaling and functional design of the cercal wind-receptor hair of cricket", J. Comp. Physiology A 183, 171-186

[18] J. Humphrey, R. Devarakonda, I. Iglesias, F. Barth, "Dynamics of arthropod filiform hairs. I. Mathematical modeling of the hair and air motions", Phil. Trans.: Bio. Sc. 340, 423-444, 1993.

[19] G. Krijnen, A. Floris, M. Dijkstra, T. Lammerink, R. Wiegerink, "Biomimetic micromechanical adaptive flow-sensor arrays", SPIE Proc. vol. 6592, paper 6592-16, 2007.

[20] R. Wiegerink, A. Floris, R. Jaganatharaja, N. Izadi, T. Lammerink, G. Krijnen, "Biomimetic Flow-Sensor Arrays Based on the Filiform Hairs on the Cerci of Crickets", Proc. IEEE Sensors 2007, Atlanta, GA, USA, in press.

[21] M. Dijkstra, J. van Baar, R. Wiegerink, T. Lammerink, J. de Boer and G. Krijnen, "Artificial sensory hairs based on the flow sensitive receptor hairs of crickets", J. Microm. Microeng. 15, S132-S138, 2005.

[22] H-E. de Bree, V.B. Svetovoy, R. Raangs, R. Visser, "The very near field theory, simulations and measurements of sound pressure and particle velocity in the very near field", 11th Int. Cong. on Sound and Vibration (ICSV11), St. Petersburg 2004.

[23] MicroChem SU-8 data-sheets see http://www.microchem.com

[24] M. Jensen, T. Weiss-Fogh, "Biology and Physics of Locust Flight. V. Strength and Elasticity of Locust Cuticle", Philos Trans R Soc Lond B, Vol 245, pg 137 - 163, 1962.

[25] G. Krijnen, et al., "MEMS based hair flow-sensors as model systems for acoustic perception studies", Nanotechnology, vol 17, pp. 84-89, 2006. 